\title{
Blood product conservation is associated with improved outcomes and reduced costs after cardiac surgery
}

\author{
Damien J. LaPar, MD, MSc, ${ }^{a}$ Ivan K. Crosby, MD, ${ }^{a}$ Gorav Ailawadi, MD, ${ }^{a}$ Niv Ad, MD, ${ }^{b}$ Elmer Choi, MD, ${ }^{b}$ \\ Bruce D. Spiess, MD, ${ }^{\mathrm{c}}$ Jeffery B. Rich, MD, ${ }^{\mathrm{d}}$ Vigneshwar Kasirajan, MD, ${ }^{\mathrm{c}}$ Edwin Fonner, Jr, DrPH, \\ Irving L. Kron, MD, ${ }^{a}$ and Alan M. Speir, MD, ${ }^{b}$ Investigators for the Virginia Cardiac Surgery Quality \\ Initiative
}

\begin{abstract}
Background: Efforts to reduce blood product use have the potential to avoid transfusion-related complications and reduce health care costs. The purpose of this investigation was to determine whether a multi-institutional effort to reduce blood product use affects postoperative events after cardiac surgical operations and to determine the influence of perioperative transfusion on risk-adjusted outcomes.
\end{abstract}

\begin{abstract}
Methods: A total of 14,259 patients (2006-2010) undergoing nonemergency, primary, isolated coronary artery bypass grafting operations at 17 different statewide cardiac centers were stratified according to transfusion guideline era: pre-guideline $(n=7059$, age $=63.7 \pm 10.6$ years $)$ versus post-guideline $(n=7200$, age $=63.7 \pm 10.5$ years). Primary outcomes of interest were observed differences in postoperative events and mortality riskadjusted associations as estimated by multiple regression analysis.
\end{abstract}

\begin{abstract}
Results: Overall intraoperative ( $24 \%$ vs $18 \%, P<.001)$ and postoperative $(39 \%$ vs $33 \%, P<.001)$ blood product transfusion were significantly reduced in the post-guideline era. Patients in the post-guideline era demonstrated reduced morbidity with decreased pneumonia $(P=.01)$, prolonged ventilation $(P=.05)$, renal failure $(P=.03)$, new-onset hemodialysis $(P=.004)$, and composite incidence of major complications $(P=.001)$. Operative mortality $(1.0 \%$ vs $1.8 \%, P<.001)$ and postoperative ventilation time ( 22 vs 26 hours, $P<.001)$ were similarly reduced in the post-guideline era. Of note, after mortality risk adjustment, operations performed in the post-guideline era were associated with a $47 \%$ reduction in the odds of death (adjusted odds ratio, 0.57; $P<.001$ ), whereas the risk of major complications and mortality were significantly increased after intraoperative (adjusted odds ratio, 1.86 and 1.25; both $P<.001$ ) and postoperative (adjusted odds ratio, 4.61 and 4.50, both $P<.001)$ transfusion. Intraoperative and postoperative transfusions were associated with increased adjusted incremental total hospitalization costs ( $\$ 4408$ and $\$ 10,479$, respectively).
\end{abstract}

Conclusions: Implementation of a blood use initiative significantly improves postoperative morbidity, mortality, and resource utilization. Limiting intraoperative and postoperative blood product transfusion decreases adverse postoperative events and reduces health care costs. Blood conservation efforts are bolstered by collaboration and guideline development. (J Thorac Cardiovasc Surg 2013;145:796-804)

Perioperative anemia is associated with increased morbidity and mortality among patients undergoing cardiac surgery, and accumulated evidence has demonstrated that allogenic blood product transfusion is an effective method of treating anemia. ${ }^{1-5}$ However, conflicting data have demonstrated that perioperative blood transfusion is associated with

\footnotetext{
From the University of Virginia, ${ }^{\text {a }}$ Charlottesville, Va; Inova Heart and Vascular Institute, ${ }^{\mathrm{b}}$ Falls Church, Va; Virginia Commonwealth University, ${ }^{\mathrm{c}}$ Richmond, Va; Mid Atlantic Cardiothoracic Surgeons, ${ }^{\mathrm{d}}$ Norfolk, Va; and Virginia Cardiac Surgery Quality Initiative, ${ }^{\mathrm{e}}$ Charlottesville, Va.

Disclosures: Authors have nothing to disclose with regard to commercial support.

Read at the 92nd Annual Meeting of The American Association for Thoracic Surgery, San Francisco, California, April 28-May 2, 2012.

Received for publication May 1, 2012; revisions received Nov 26, 2012; accepted for publication Dec 11, 2012.

Address for reprints: Damien J. LaPar, MD, MSc, Department of Surgery, University of Virginia, PO Box 800681, Charlottesville, VA 22908 (E-mail: dj18w@ virginia.edu). $0022-5223 / \$ 36.00$

Copyright (c) 2013 by The American Association for Thoracic Surgery http://dx.doi.org/10.1016/j.jtcvs.2012.12.041
}

greater postoperative complications, including nosocomial infections, immunosuppression, transfusion-related acute lung injury, decreased health-related quality of life, and reduced early and long-term survival. ${ }^{6-18}$ As a result, efforts to reduce blood product use have the potential to avoid transfusion-related complications and to reduce health care costs. To date, many series investigating strategic approaches to blood conservation in cardiac surgery are limited by single institutional experiences. ${ }^{11,19-23}$ Few studies have investigated the economic considerations of blood transfusion-related costs and the potential cost-savings of a successful blood conservation initiative.

The Virginia Cardiac Surgery Quality Initiative (VCSQI) is a voluntary consortium of 17 different collaborating cardiac surgical centers, both academic and private, within the Commonwealth of Virginia. This group exchanges and compares deidentified patient data on a quarterly basis in an effort to improve cardiac surgical care, quality, and costs. 

Abbreviations and Acronyms
$\mathrm{AOR}=$ adjusted odds ratio
AUC $=$ area under the receiver operating characteristic curve
$\mathrm{CABG}=$ coronary artery bypass grafting
HGLM $=$ hierarchic general linear model
PRBC $=$ packed red blood cell
$\mathrm{PROM}=$ predicted risk of mortality
SCIP = Surgical Care Improvement
STS = Society of Thoracic Surgeons
VCSQI $=$ Virginia Cardiac Surgery Quality Initiative

The primary objective of the organization is to identify quality-improvement opportunities in cases in which high-cost, resource-intensive, or frequently occurring preventable outcomes might occur. Collectively, the VCSQI centers perform approximately $99 \%$ of the Commonwealth's cardiac operations, and each center individually contributes patient data to the national Society of Thoracic Surgeons (STS) Adult Cardiac Surgery Database.

The purpose of this study was (1) to determine whether a multi-institutional effort to reduce blood product transfusion could be successfully implemented and achieved, and (2) to assess the impact of established transfusion guidelines on postoperative events after cardiac surgical operations and the influence of intraoperative and postoperative blood product transfusion on risk-adjusted outcomes. We hypothesized that successful implementation of blood product transfusion guidelines would result in a significant reduction in intraoperative and postoperative blood product transfusion and improve patient outcomes.

\section{MATERIALS AND METHODS}

This investigation was exempt from formal institutional review board review at each participating center because it represents a secondary analysis of the VCSQI data registry with the absence of Health Insurance Portability and Accountability Act patient identifiers and because the data are collected for quality analysis and purposes other than research.

\section{Patients and Data Acquisition}

Deidentified patient-level data were obtained from the VCSQI database for the study period August 1, 2006, to June 30, 2010. All records included patients undergoing nonemergency, isolated, primary coronary artery bypass grafting (CABG) operations (STS procedure type "CAB Only"). Patient records were stratified according to transfusion guideline era: pre-guideline era (August 1, 2008, to July 31, 2008) and postguideline era (August 1, 2008, to July, 31, 2010). Emergency CABG cases were excluded. All CABG procedures represent standard surgical approaches to surgical myocardial revascularization with and without the use of cardiopulmonary bypass support, and standard STS variable definitions were used. ${ }^{24}$ Patient preoperative risk was assessed by prevalence of patient comorbid disease, extent of coronary artery disease, operative status, and individual calculated STS predicted risk of mortality (PROM).

\section{Blood Product Use Protocol}

The potential clinical and financial benefits that could be achieved through a coordinated blood conservation initiative represents one of the leading efforts by the VCSQI since early 2008. Among VCSQI participants, consensus agreement was achieved to establish a blood conservation initiative with blood product transfusion guidelines to be implemented for a consecutive 2-year period beginning in August 2008. The adopted transfusion guidelines included both intraoperative and postoperative transfusion thresholds and practices of care. Intraoperative transfusion triggers included packed red blood cell (PRBC) transfusion for hemoglobin levels less than $6.0 \mathrm{~g} / \mathrm{dL}$ or a hematocrit level less than $18 \%$ while on cardiopulmonary bypass in addition to one of the following: (1) low oxygen saturation $(<60 \mathrm{~mm}$ $\mathrm{Hg}$ ), (2) elevated lactate level ( $>2.2 \mathrm{mmol} / \mathrm{L}$ ), (3) elevated base deficit (base deficit $>3 \mathrm{mEq} / \mathrm{L}$ ), or (4) low serum bicarbonate level $(<22 \mathrm{mEq} / \mathrm{L})$. Similar postoperative transfusion triggers also were established to include PRBC transfusion for hemoglobin levels less than $7.0 \mathrm{~g} / \mathrm{dL}$ or hematocrit level less than $21 \%$ in addition to at least one of the following clinical criteria: (1) elevated oxygen requirement, (2) systemic hypotension, (3) evidence of end-organ dysfunction, or (4) evidence of persistent bleeding.

\section{Measured Outcomes}

The primary outcomes of interest included risk-adjusted associations between mortality and morbidity and transfusion guideline era, and intraoperative and postoperative blood product transfusion. Secondary outcomes included estimated risk-adjusted correlations between intraoperative and postoperative blood product transfusion and total costs and observed differences in the incidence of postoperative events. Operative mortality was defined as all patient deaths occurring during hospitalization and those within 30 days of the date of surgery despite discharge status. A composite outcome of major complications was used as a proxy for major morbidity and included the incidence of deep sternal wound infection, postoperative stroke, renal failure, prolonged ventilation, pneumonia, and need for reoperation. Standard STS definitions for postoperative events and complications were used, including prolonged ventilation ( $>24$ hours of mechanical ventilation), presence of any new-onset atrial fibrillation, and renal failure (increase in serum creatinine level $>2.0$ or a doubling $[2 \times]$ of the most recent preoperative creatinine level). ${ }^{24}$

\section{Cost Data and Acquisition}

VCSQI uses an information system that combines standardized clinical data extracted from STS data-entry forms with hospital inpatient discharge financial data. Hospital inpatient data from UB-92 and UB-04 files are matched with each STS patient record. The patient data involve inpatient hospital stays exclusively for cardiac surgical operations. The UB-92 and UB-04 file contains the final bill (not cyclic or interim bills) and the patient's date of discharge, and is accompanied by a charge detail file and a physician file. Considering that the UB-92 and UB-04 records contain patient charge data, the VCSQI developed a 2-step procedure making the financial data more comparable from one provider organization to another. First, all of the International Classification of Diseases, 9th Revision-based revenue codes contained in the patient records are aggregated and sorted into 21 different cost categories. Second, a set of cost-to-charge ratios is submitted from each provider organization and multiplied by each of the 21 cost categories. The product is a set of normalized charges that are approximately comparable from one surgeon and provider to another. When the normalized charges are summed over the 21 cost categories, an estimate of the total cost of that inpatient stay is derived. The VCSQI maintains a $99 \%$ matching rate between STS patient records and billing data.

\section{Statistical Analysis}

All statistical analyses were designed to test the null hypotheses that patient outcomes would not significantly differ as a function of transfusion guideline era or the presence of intraoperative/postoperative blood product 
transfusion. Study outcomes and data comparisons were established a priori before data collection. Categoric variables are expressed as standard group percentages, and continuous variables are expressed as mean \pm standard deviation or median (25th, 75 th percentile) depending on overall variable distribution. Descriptive, univariate statistics included Pearson's chi-square or Fisher exact test for categoric variables and the independent sample single factor analysis of variance for comparisons of normally distributed data or the Mann-Whitney $U$ test for non-normally distributed data comparisons. Calculated test statistics were used to derive all 2 -tailed $P$ values.

Hierarchic multiple logistic regression models were used to estimate confounder-adjusted associations between intraoperative and postoperative blood product transfusion, and transfusion guideline era and observed patient morbidity (major complications) and operative mortality. To account for inter-hospital variance in correlated events, clustering at the hospital level was considered in the hierarchic structure of each logistic regression model. Model covariates were selected a priori on the basis of established clinical risk or considered potential confounders for the influence of blood product protocol implementation on patient outcomes. Performed models were adjusted for baseline patient risk by inclusion of the calculated STS PROM score, individual surgeon influence on patient outcomes and transfusion behavior by inclusion of surgeon identification, influence of baseline changes in practice over the study period by inclusion of the operative/ transfusion era, and intraoperative and postoperative blood product transfusion. In addition, a hierarchic general linear model (HGLM) was used to assess the risk-adjusted influence of intraoperative and postoperative blood product transfusion on total costs, adjusting for the same model covariates. Confounder-adjusted measures of association are reported as odds ratios with a $95 \%$ confidence interval for all logistic regression models and the unstandardized $\beta$ with a $95 \%$ confidence interval for HGLM results. Model performance was assessed using the area under the receiver operating characteristic curve (AUC), and the Hosmer-Lemeshow test was used to verify model calibration across deciles of observed and predicted risk. Predictive Analytics SoftWare (PASW) with complex sampling module software, version 18.0.0 (IBM Corp, Somers, NY) was used for all data manipulation and statistical analyses.

\section{RESULTS}

\section{Patient Characteristics and Operative Features for Coronary Artery Bypass Grafting}

Table 1 reports patient characteristics and operative features for all patients undergoing $\mathrm{CABG}$ operations stratified by blood product transfusion guideline era. A total of 7059 patients $(49.5 \%)$ underwent CABG during the preguideline era (August 2006 to July 2008), and 7200 patients $(50.5 \%)$ underwent $\mathrm{CABG}$ during the post-guideline era (August 2008 to July 2010). The average patient age was 63 years in both surgical eras, and gender distribution was the same. With respect to major cardiovascular comorbidities, small differences were prevalent between patient cohorts. Patients in the pre-guideline era presented with slightly lower percentage of New York Heart Association class III and IV functional status, slightly higher percentage of preoperative renal failure, and lower percentage of prior percutaneous coronary interventions. Small differences in the type of cardiac presentation of admission existed between groups. The presence of significant left main coronary disease was approximately $30 \%$ in each patient cohort. Patients in the post-guideline era presented with a higher percentage of preoperative aspirin (acetylsalicylic
TABLE 1. Descriptive statistics for patients undergoing coronary artery bypass grafting by transfusion guideline era

\begin{tabular}{|c|c|c|c|}
\hline Variable & $\begin{array}{l}\text { Pre-guideline } \\
\text { era }(n=7059)\end{array}$ & $\begin{array}{l}\text { Post-guideline } \\
\text { era }(n=7200)\end{array}$ & $P$ \\
\hline Age $(y)^{*}$ & $63.7 \pm 10.6$ & $63.7 \pm 10.5$ & .97 \\
\hline Sex (female) & $26.0 \%$ & $26.0 \%$ & .37 \\
\hline Hypertension & $81.7 \%$ & $85.1 \%$ & $<.001$ \\
\hline Diabetes & $39.8 \%$ & $39.8 \%$ & .97 \\
\hline Dyslipidemia & $72.2 \%$ & $71.4 \%$ & .30 \\
\hline Atrial fibrillation & $3.7 \%$ & $4.1 \%$ & .22 \\
\hline PVD & $13.5 \%$ & $13.0 \%$ & .42 \\
\hline NYHA class & & & .02 \\
\hline I & $7.6 \%$ & $5.1 \%$ & \\
\hline II & $35.3 \%$ & $34.2 \%$ & \\
\hline III & $42.5 \%$ & $43.7 \%$ & \\
\hline IV & $14.5 \%$ & $17.0 \%$ & \\
\hline Renal failure & $4.0 \%$ & $3.1 \%$ & $<.001$ \\
\hline $\begin{array}{l}\text { Cardiac presentation on } \\
\text { admission }\end{array}$ & & & $<.001$ \\
\hline No symptoms or angina & $9.4 \%$ & $9.1 \%$ & \\
\hline Stable angina & $26.2 \%$ & $26.7 \%$ & \\
\hline Unstable angina & $34.6 \%$ & $35.3 \%$ & \\
\hline ST-elevation MI & $6.3 \%$ & $6.0 \%$ & \\
\hline Non-ST-elevation MI & $21.0 \%$ & $20.8 \%$ & \\
\hline Prior PCI & $21.4 \%$ & $23.3 \%$ & .008 \\
\hline Left main $>50 \%$ & $30.5 \%$ & $29.6 \%$ & .21 \\
\hline Preoperative ASA & $83.0 \%$ & $87.3 \%$ & $<.001$ \\
\hline Antiplatelet within $5 \mathrm{~d}$ & $1.6 \%$ & $4.2 \%$ & $<.001$ \\
\hline STS PROM $(\%) \dagger$ & $0.9 \%(0.6-1.8)$ & $0.8 \%(0.4-1.6)$ & $<.001$ \\
\hline
\end{tabular}

$P V D$, Peripheral vascular disease; $N Y H A$, New York Heart Association; $M I$, myocardial infarction; $P C I$, percutaneous coronary intervention; $A S A$, acetylsalicylic acid; STS, Society of Thoracic Surgeons; PROM, predicted risk of mortality; ST, segment. *Results reported as mean \pm standard deviation. $\dagger$ Results reported as median (25th75 th percentile).

acid) use and antiplatelet therapy (clopidogrel) within 5 days of surgery compared with those in the pre-guideline era. Ultimately, these differences translated into minor differences in preoperative risk as assessed by calculated STS PROM scores, with patients in the pre-guideline era presenting with median PROM scores of $0.9 \%$ compared with $0.8 \%$ for those in the post-guideline era $(P<.001)$.

Table 2 displays the operative details for patients undergoing CABG in both pre- and post-guideline eras. The large majority of operations were performed with the use of cardiopulmonary bypass support $(91 \%)$, with approximately $9 \%$ performed off-pump. Patients in the pre-guideline era underwent a higher percentage of elective operations, and patients in the post-guideline era underwent more urgent operations. The majority of revascularizations included use of the left internal thoracic artery and a single saphenous vein graft.

\section{Comparison of Blood Product Transfusion Rates by Guideline Era}

The incidence of intraoperative and postoperative blood product transfusion, and differences in transfused products 
TABLE 2. Operative features of patients undergoing coronary artery bypass grafting procedures by transfusion guideline era

\begin{tabular}{lccc}
\hline \multicolumn{1}{c}{ Outcome } & $\begin{array}{c}\text { Pre-guideline } \\
\text { era }(\mathbf{n = 7 0 5 9})\end{array}$ & $\begin{array}{r}\text { Post-guideline } \\
\text { era }(\mathbf{n = 7 2 0 0})\end{array}$ & $\boldsymbol{P}$ \\
\hline CPB & $92.5 \%$ & $90.6 \%$ & $<.001$ \\
Elective status & $48.2 \%$ & $37.7 \%$ & $<.001$ \\
Urgent status & $51.8 \%$ & $62.7 \%$ & $<.001$ \\
SVG & & & $<.001$ \\
1 SVG & $85.2 \%$ & $84.8 \%$ & \\
2 SVG & $8.6 \%$ & $8.9 \%$ & \\
3 SVG & $1.6 \%$ & $2.0 \%$ & \\
$\geq 4$ SVGs & $0.3 \%$ & $0.7 \%$ & \\
LITA & $91.3 \%$ & $93.2 \%$ & $<.001$ \\
RITA & $0.3 \%$ & $0.4 \%$ & \\
BITA & $4.0 \%$ & $2.7 \%$ & \\
\hline
\end{tabular}

$C P B$, Cardiopulmonary bypass; $S V G$, saphenous vein graft; $L I T A$, left internal thoracic artery; RITA, right internal thoracic artery; BITA, bilateral internal thoracic artery.

between transfusion guideline eras are detailed in Table 3 . Overall, significant decreases in the rate of both intraoperative and postoperative transfusion were observed in the postguideline era. Intraoperative transfusion rates decreased by $7 \%(24 \%$ vs $17 \%, P<.001)$ in the post-guideline era. Likewise, postoperative transfusion rates decreased by $6 \%(39 \%$ vs $33 \%, P<.001)$ in the post-guideline era. With respect to specific blood components, the average number of total transfused intraoperative products and intraoperative PRBC units significantly decreased in the post-guideline era. Of note, a significant reduction in the average number of postoperative transfusions (total units, PRBC units, fresh-frozen plasma units, and platelet units) also was observed in the post-guideline era.

\section{Comparisons of Unadjusted Incidence of Postoperative Events as Function of Transfusion Guideline Era}

Significant improvement in the unadjusted incidence of postoperative morbidity and mortality was observed during

TABLE 3. Comparison of blood product use by transfusion guideline era

\begin{tabular}{lccc}
\hline \multicolumn{1}{c}{ Outcome } & $\begin{array}{c}\text { Pre-guideline } \\
\text { era }(\mathbf{n}=\mathbf{7 0 5 9})\end{array}$ & $\begin{array}{c}\text { Post-guideline } \\
\text { era }(\mathbf{n}=\mathbf{7 2 0 0})\end{array}$ & $\boldsymbol{P}$ \\
\hline Intraoperative transfusion & $24.0 \%$ & $17.7 \%$ & $<.001$ \\
Total products $(\mathrm{U}) *$ & $0.6 \pm 1.8$ & $0.1 \pm 1.5$ & $<.001$ \\
PRBC (U)* & $0.4 \pm 0.8$ & $0.2 \pm 0.8$ & $<.001$ \\
FFP (U)* & $0.1 \pm 0.2$ & $0.1 \pm 0.3$ & .30 \\
Platelet (U)* & $0.2 \pm 1.0$ & $0.1 \pm 0.6$ & .07 \\
Postoperative transfusion & $39.1 \%$ & $32.8 \%$ & $<.001$ \\
Total products (U)* & $1.4 \pm 3.8$ & $0.4 \pm 1.9$ & $<.001$ \\
PRBC (U)* & $0.9 \pm 2.0$ & $0.7 \pm 1.9$ & $<.001$ \\
FFP (U)* & $0.2 \pm 0.9$ & $0.1 \pm 1.1$ & $<.001$ \\
Platelet (U)* & $0.2 \pm 1.2$ & $0.1 \pm 0.6$ & $<.001$ \\
\hline PRBC, Packed
\end{tabular}

PRBC, Packed red blood cells; FFP, fresh-frozen plasma. *Results reported as mean \pm standard deviation. the post-guideline era (Table 4). Although statistically shorter in the post-guideline era, median cardiopulmonary bypass times were clinically similar during both transfusion eras. With respect to postoperative morbidity, patients in the post-guideline era incurred lower composite major complication rates $(13 \%$ vs $15 \%, P<.001)$ with decreased incidence of pneumonia, prolonged ventilation, renal failure, and new-onset hemodialysis requirements. Postoperative ventilation time was similarly reduced in the post-guideline era. Blood conservation also resulted in a significant decrease in operative mortality during the post-guideline era $(1.0 \%)$ compared with pre-guideline era mortality $(1.8 \%, P<.001)$. Despite similar postoperative hospital durations (median, 5 days), patients in the post-guideline era accrued significantly lower total costs than patients in the pre-guideline era. Overall total hospitalization costs for the patients in the pre-guideline era was \$261 million compared with \$212 million for patients in the post-guideline era, providing for a net decrease in total costs of $\$ 49$ million $(P<.001)$. Likewise, a median reduction of approximately $\$ 4000$ per patient hospitalization $(P<.001)$ was observed during the post-guideline era. These cost-savings were largely driven by reductions in intensive care unit/cardiac care unit costs $(P<.0001)$ and respiratory therapy costs $(P<.0001)$ among patients in the post-guideline era.

\section{Results of Risk-Adjusted Models}

Because of differences in patient risk profiles, riskadjusted associations between transfusion guideline era and as intraoperative and postoperative blood product transfusions and patient morbidity and mortality were estimated using hierarchic multiple regression models (Table 5). After adjusting for the influence of hospital variability and patient risk, using the calculated STS PROM, CABG operations performed in the post-guideline era were associated with a $47 \%$ reduction in the odds of mortality (adjusted odds ratio [AOR], 0.53; $P<.001)$ compared with operations performed in the pre-guideline era. On estimating the influence of blood product transfusion on morbidity and mortality, intraoperative transfusion was associated with a $25 \%$ increase in the likelihood of major complications (AOR, $1.25 ; P<.001)$ and an $86 \%$ increase in the likelihood of mortality (AOR, 1.86; $P<.001$ ). The estimated impact of postoperative transfusion was even more profound, with transfusion of blood products associated with 4.5 -fold and 4.6-fold increases in the odds of major complications (AOR, 4.50; $P<.001$ ) and mortality (AOR, 4.61; $P<.001)$ after risk factor adjustment. With respect to the influence of product transfusion on total costs, HGLM results demonstrated positive correlations between the transfusion of intraoperative $(B=+4408, P<.001)$ and postoperative $(B=+10,479, P<.001)$ blood products and total hospitalization costs. Thus, the incremental cost 
TABLE 4. Unadjusted patient outcomes by transfusion guideline era

\begin{tabular}{|c|c|c|c|}
\hline Outcome & Pre-guideline era $(n=7059)$ & Post-guideline era $(n=7200)$ & $\boldsymbol{P}$ \\
\hline Crossclamp time $(\min )^{*}$ & $65(50-82)$ & $66(51-84)$ & $<.001$ \\
\hline Bypass time $(\min )^{*}$ & $92(73-115)$ & $91(72-115)$ & $<.001$ \\
\hline Reoperation for bleeding/tamponade & $2.1 \%$ & $1.2 \%$ & $<.001$ \\
\hline Stroke & $1.3 \%$ & $1.3 \%$ & $>.99$ \\
\hline Perioperative MI & $0.2 \%$ & $0.1 \%$ & .32 \\
\hline Pneumonia & $2.7 \%$ & $2.0 \%$ & .01 \\
\hline Prolonged ventilation & $4.7 \%$ & $4.4 \%$ & .05 \\
\hline Renal failure & $3.8 \%$ & $3.1 \%$ & .03 \\
\hline Hemodialysis & $1.5 \%$ & $1.0 \%$ & .004 \\
\hline Major complication & $15.1 \%$ & $13.2 \%$ & .001 \\
\hline Postoperative ventilation time $(\mathrm{h})^{*}$ & $26(5-16)$ & $22(5-13)$ & $<.001$ \\
\hline Operative mortality & $1.8 \%$ & $1.0 \%$ & $<.001$ \\
\hline Postoperative LOS $(\mathrm{d})^{*}$ & $5(4-7)$ & $5(4-7)$ & .97 \\
\hline Median total hospitalization costs $(\$)^{*}$ & $\$ 30,487(24,516-39,111)$ & $\$ 26,197(21,557-32,752)$ & $<.001$ \\
\hline Median ICU/CCU costs $(\$)^{*}$ & $\$ 6355(4033-10,112)$ & $\$ 5,710(3413-8478)$ & $<.001$ \\
\hline Median respiratory therapy costs $(\$)^{*}$ & $\$ 482(273-797)$ & $\$ 425(240-730)$ & $<.001$ \\
\hline
\end{tabular}

$M I$, Myocardial infarction; $L O S$, length of stay; $I C U$, intensive care unit; $C C U$, critical care unit. *Results reported as median (25th-75th percentile).

per hospitalization associated with intraoperative blood product transfusion after mortality risk adjustment was \$4408, whereas the adjusted incremental cost per hospitalization associated with postoperative transfusion of blood products was $\$ 10,479$.

The statistical performance of each logistic regression model achieved good discrimination with an AUC of 0.84 and 0.76 for the operative mortality model and major complications model, respectively. AUC values of 1.0 indicate perfect model discrimination between dependent outcomes, and AUC values of 0.5 represent discrimination equal to chance. The calibration of each model was adequate across deciles of observed risk as reflected by a HosmerLemeshow $P$ value less than .05 for both models.

\section{DISCUSSION}

The present study reports the successful implementation of a coordinated, multi-institutional blood conservation initiative within the Commonwealth of Virginia. In this

TABLE 5. Results of mortality risk-adjusted hierarchic regression models for effect of operative era and blood product transfusion on patient outcomes

\begin{tabular}{lccc}
\hline \multicolumn{1}{c}{ Factor } & OR or $\boldsymbol{B}$ & $\mathbf{9 5} \% \mathbf{C I}$ & $\boldsymbol{P}$ \\
\hline Operative era & & & \\
$\quad$ Major complications & 0.97 & $0.88-1.08$ & .64 \\
$\quad$ Mortality & 0.53 & $0.37-0.74$ & $<.001$ \\
Intraoperative transfusion & & & \\
$\quad$ Major complications & 1.25 & $1.12-1.41$ & $<.001$ \\
$\quad$ Mortality & 1.86 & $1.35-2.55$ & $<.001$ \\
$\quad$ Total costs $(\$)$ & +4408 & $3337-5480$ & $<.001$ \\
Postoperative transfusion & & & \\
$\quad$ Major complications & 4.50 & $4.03-5.03$ & $<.001$ \\
$\quad$ Mortality & 4.61 & $3.19-6.68$ & $<.001$ \\
$\quad$ Total costs $(\$)$ & $+10,479$ & $9581-11,376$ & $<.001$ \\
\hline OR, & & &
\end{tabular}

$O R$, Odds ratio; $C I$, confidence interval. contemporary analysis of nonemergency, isolated, primary CABG outcomes, implementation of transfusion guidelines resulted in a significant decrease in patient morbidity, mortality, and healthcare costs. Specifically, adoption of transfusion guidelines resulted in decreased intraoperative and postoperative blood product transfusion rates and a reduction in the amount of products transfused in both clinical settings. As a result, patients in the post-guideline era incurred important reductions in composite major complication rates and operative mortality, as well as the accrual of lower total hospitalization costs. The substantial benefits derived from the initiation of blood product transfusion guidelines was even more apparent after mortality risk adjustment with an estimated $47 \%$ reduction in the odds of mortality associated with operations performed in the post-guideline era. These results also quantify the riskadjusted impact of intraoperative and postoperative blood product transfusion on postoperative morbidity and mortality, demonstrating the significant risk associated with blood product transfusion in both settings. Unique in the present findings was the demonstration of the financial implications associated with blood product transfusion and the significant reductions in healthcare costs that could be achieved through a multi-institutional commitment to transfusion restraint. To our knowledge, these data represent the only series to specifically report on a statewide, multi-institutional cardiac surgery blood conservation effort to demonstrate both the clinical and the economic benefits of decreased blood product use for CABG operations.

A primary objective of the reported initiative was to establish whether predefined blood conservation guidelines could be successfully implemented at a multi-institutional level to effectively reduce intraoperative and postoperative transfusion rates. The reported results demonstrate that the blood conservation program was effective in reducing 
overall transfusion rates in the post-guideline era among 17 different cardiac centers. These reductions demonstrate that it is possible to significantly reduce transfusion practices and rates in cardiac surgery with coordinated, multidisciplinary programs that effectively manage permissive anemia in the intraoperative and postoperative settings. The reported reductions in transfusion rates compare favorably to other reported single institution series, which further highlights the success of the coordinated efforts between multiple institutions in the present results. In one prospective, randomized trial of patients undergoing CABG, Bracey and colleagues ${ }^{25}$ reported postoperative transfusion rates of $20 \%$ using a transfusion threshold of hemoglobin less than $8 \mathrm{~g} / \mathrm{dL}$ without significant differences in postoperative morbidity or mortality. In 2 other related series, DeAnda and colleagues ${ }^{26}$ and Moskowitz and colleagues ${ }^{22}$ demonstrated substantial reductions in postoperative transfusion rates of $16 \%$ and $11 \%$, respectively, at single institutions after implementation of extensive blood conservation programs. Although these series demonstrate excellent success at the single-institution level, these and other prior published reports have failed to demonstrate that significant change in transfusion practices can occur at a larger statewide or regional level.

The principle findings of reduced morbidity, mortality, and associated risks resulting from adoption of blood conservation guidelines in the present series demonstrate the safety and efficacy of this multi-institutional approach to a patient quality initiative and are in agreement with other published benefits of blood conservation in cardiac surgery. Instrumental to the initiation and development of an evidence-based approach to the VCSQI blood conservation guidelines were the STS and the Society of Cardiovascular Anesthesiologists clinical practice guidelines for perioperative blood transfusion and conservation in cardiac surgery. ${ }^{27}$ Moreover, several series have demonstrated similar reductions in morbidity associated with blood conservation programs and restrictive transfusion strategies. ${ }^{22,28}$ Hebert and colleagues ${ }^{28}$ reported on the safety of a restrictive transfusion strategy (hemoglobin 7-9 g/dL) compared with a liberal strategy (hemoglobin 10-12 $\mathrm{g} / \mathrm{dL}$ ) in a prospectively randomized study of 848 patients and demonstrated significant differences in mortality between study groups. Another recent study of 2 propensity-matched study cohorts of 586 patients reported that a blood conservation program resulted in significantly lower serious $(11 \%)$ and very serious complications $(10.6 \%)$ after $\mathrm{CABG} .{ }^{22}$ In this series, the $13 \%$ major complication rate reported among patients in the post-guideline era coupled with the $1 \%$ mortality rate for this cohort is consistent with these reports and supports a restrictive transfusion strategy. More important, the benefits of restrictive transfusion guidelines were even more apparent after mortality and hospital risk adjustment. After mortality risk adjustment, patients undergoing operations since the introduction of transfusion guidelines were associated with a $47 \%$ reduced likelihood of death compared with patients undergoing the same operations before established guidelines. Coupled with the aforementioned reductions in observed morbidity, these results provide an important foundation from which future randomized controlled trials using these transfusion guideline triggers can be designed.

Transfusion of both intraoperative and postoperative blood products was significantly associated with increased mortality and morbidity in this series regardless of blood conservation strategy or guideline implementation. On hierarchic logistic regression modeling, intraoperative transfusion was associated with a $25 \%$ increase in the odds of composite major complications (AOR, 1.25; $P<.001)$ and an $86 \%$ increase in the odds of mortality (AOR, 1.86; $P<.001$ ). Even more striking was that postoperative transfusion was associated with a 4.5 -fold increase $(P<.001)$ in the odds of composite major complications and a 4.6 -fold increase $(P<.001)$ in mortality. It is possible that the influence of significant postoperative bleeding may have affected these estimated effects; however, because of the relatively small proportion of patients undergoing reoperations for bleeding/tamponade ( $<2 \%$ overall), we believe this influence to be small. Nevertheless, the observed associations between transfusions and patient morbidity and mortality in this series are well corroborated in other reports. ${ }^{7,9-12}$ Koch and colleagues ${ }^{9}$ reported on more than 10,000 patients undergoing $\mathrm{CABG}$ and demonstrated that transfusion was associated with reduced short- and long-term risk-adjusted survival. Likewise, Whitson and colleagues ${ }^{29}$ identified transfusion as an independent risk factor for increased morbidity and mortality among patients undergoing cardiac surgery. Moreover, Surgenor and coauthors ${ }^{12}$ demonstrated that exposure to 1 or 2 units of transfused PRBC was associated with a $16 \%$ hazard of decreased survival in a multiinstitutional cohort of more than 9000 patients. Thus, the present results serve to bolster those of previous reports to further advocate for coordinated blood conservation programs to further reduce cardiac surgical risk and morbidity.

A unique aspect of this report are the findings of significant cost reductions that occurred with the successful implementation of a blood conservation initiative. The striking findings that total hospitalization costs were reduced by $\$ 49$ million dollars statewide over a 2-year study period with a median reduction of $\$ 4000$ per patient hospitalization after the introduction of the blood conservation guidelines demonstrate the important financial implications that coordinated quality efforts can achieve. Moreover, the financial impact of blood product transfusion and the reported $\$ 4408$ and $\$ 10,479$ adjusted incremental costs of hospitalization associated with intraoperative and postoperative transfusion, respectively, are in agreement with other reported cost analyses. In a report examining the financial impact of blood product transfusions on hospital costs 
among inpatient surgical patients, Stokes and colleagues ${ }^{30}$ reported that the incremental cost per hospitalization associated with bleeding-related complications or transfusions was $\$ 10,279$ among a total of 103,829 patients undergoing cardiac surgery. The authors noted similar associations between blood transfusion and hospitalization costs for vascular and noncardiac thoracic surgical populations. ${ }^{30}$

The reported results have important clinical implications. In an era of increasingly complex operations and patients, these results provide clinical estimates of the adjusted impact of blood conservation guidelines on the performance of CABG operations. Moreover, these results represent a large, multi-institutional cohort of patients, providing a report of outcomes that are more generalizable than those of other small, single-institutional series. The use of multilevel structured multivariable regression models with strong performance indices demonstrates that the granularity of the analyzed data is appropriately controlled for in the reported statistical associations. Most important, these results highlight the importance of cooperative regional efforts in the recognition of important quality-improvement opportunities and guideline development to derive both clinical and financial benefits for patients undergoing cardiac surgery.

\section{Study Limitations}

The present study has limitations. First, the secondary analysis of the VCSQI data registry and STS data limited the performed analyses to deidentified data, which did not allow for further investigation of certain data, including details related to postoperative bleeding episodes, nature of stored blood products, or use of red blood cell salvage. In fact, the influence of red blood cell storage duration has become increasingly emphasized and is the focus of the ongoing large multicenter RECESS trial supported by the National Institutes of Health. The retrospective study design is subject to limitations of inherent selection bias, and the reported results are limited to describe observed associations between the implementation of the described protocol and the improved patient outcomes and do not demonstrate a direct cause-and-effect relationship. Differences in adherence rates among participating centers to blood conservation guidelines and differences in physician behavior on transfusion practices could exert unmeasured influences in these analyses. Despite consensus agreement on guidelines, individual centers and physicians were responsible to enforce guideline practices, and the influence of less adherent centers or physicians would have biased the results toward the null hypothesis. Nevertheless, the reported results were adjusted for the confounding influence of center and physician differences; however, despite these efforts, the influence of physician behavior that has occurred over the study period cannot be completely accounted for in the reported results. Thus, the reported results have the potential to underestimate additional benefits that could be achieved with expanded implementation. The influence of differences in postoperative critical care management between institutions also cannot be fully accounted for in these analyses; however, the hierarchic structure of the performed regression analyses likely mitigated this influence because it was designed to account for variance in correlated events between hospitals. All analyses were limited to short-term operative outcomes, and intermediate or long-term follow-up data were not available. Finally, the potential for unrecognized miscoding of data must be considered in any secondary analysis of a data registry.

\section{CONCLUSIONS}

The results of the present study demonstrate that implementation of a coordinated blood use initiative at a statewide level can significantly improve postoperative morbidity, mortality, and resource use among patients undergoing CABG operations. Limiting intraoperative and postoperative blood product transfusion decreases adverse postoperative events and reduces hospitalization-related costs. These results argue for increased blood conservation efforts bolstered by collaboration and guideline development.

The authors thank George J. Stukenborg, PhD, for statistical mentorship in this study.

\section{References}

1. Carson JL, Duff A, Poses RM, Berlin JA, Spence RK, Trout R, et al. Effect of anaemia and cardiovascular disease on surgical mortality and morbidity. Lancet. 1996;348:1055-60.

2. DeFoe GR, Ross CS, Olmstead EM, Surgenor SD, Fillinger MP, Groom RC, et al. Lowest hematocrit on bypass and adverse outcomes associated with coronary artery bypass grafting. Northern New England Cardiovascular Disease Study Group. Ann Thorac Surg. 2001;71:769-76.

3. Karkouti K, Djaiani G, Borger MA, Beattie WS, Fedorko L, Wijeysundera D, et al. Low hematocrit during cardiopulmonary bypass is associated with increased risk of perioperative stroke in cardiac surgery. Ann Thorac Surg. 2005; 80:1381-7.

4. Kulier A. Anemia and morbidity and mortality in coronary bypass surgery. Curr Opin Anaesthesiol. 2007;20:57-64.

5. Kulier A, Levin J, Moser R, Rumpold-Seitlinger G, Tudor IC, Snyder-Ramos SA, et al. Impact of preoperative anemia on outcome in patients undergoing coronary artery bypass graft surgery. Circulation. 2007;116:471-9.

6. Banbury MK, Brizzio ME, Rajeswaran J, Lytle BW, Blackstone EH. Transfusion increases the risk of postoperative infection after cardiovascular surgery. $\mathrm{J} \mathrm{Am}$ Coll Surg. 2006;202:131-8.

7. Engoren MC, Habib RH, Zacharias A, Schwann TA, Riordan CJ, Durham SJ. Effect of blood transfusion on long-term survival after cardiac operation. Ann Thorac Surg. 2002;74:1180-6.

8. Koch CG, Li L, Duncan AI, Mihaljevic T, Cosgrove DM, Loop FD, et al. Morbidity and mortality risk associated with red blood cell and blood-component transfusion in isolated coronary artery bypass grafting. Crit Care Med. 2006; 34:1608-16.

9. Koch CG, Li L, Duncan AI, Mihaljevic T, Loop FD, Starr NJ, et al. Transfusion in coronary artery bypass grafting is associated with reduced long-term survival. Ann Thorac Surg. 2006;81:1650-7.

10. Murphy GJ, Reeves BC, Rogers CA, Rizvi SI, Culliford L, Angelini GD. Increased mortality, postoperative morbidity, and cost after red blood cell transfusion in patients having cardiac surgery. Circulation. 2007;116:2544-52.

11. Scott BH, Seifert FC, Grimson R. Blood transfusion is associated with increased resource utilisation, morbidity and mortality in cardiac surgery. Ann Card Anaesth. 2008;11:15-9. 
12. Surgenor SD, DeFoe GR, Fillinger MP, Likosky DS, Groom RC, Clark C, et al. Intraoperative red blood cell transfusion during coronary artery bypass graft surgery increases the risk of postoperative low-output heart failure. Circulation. 2006;114(1 Suppl):I43-8.

13. Surgenor SD, Kramer RS, Olmstead EM, Ross CS, Sellke FW, Likosky DS, et al. The association of perioperative red blood cell transfusions and decreased longterm survival after cardiac surgery. Anesth Analg. 2009;108:1741-6.

14. Rogers MA, Blumberg N, Saint S, Langa KM, Nallamothu BK. Hospital variation in transfusion and infection after cardiac surgery: a cohort study. BMC Med. 2009;7:37.

15. Spiess BD. Blood transfusion: the silent epidemic. Ann Thorac Surg. 2001;72: S1832-7.

16. Taylor RW, O'Brien J, Trottier SJ, Manganaro L, Cytron M, Lesko MF, et al. Red blood cell transfusions and nosocomial infections in critically ill patients. Crit Care Med. 2006;34:2302-8; quiz 09

17. Toy P, Lowell C. TRALI-definition, mechanisms, incidence and clinical relevance. Best Pract Res Clin Anaesthesiol. 2007;21:183-93.

18. Koch CG, Khandwala F, Li L, Estafanous FG, Loop FD, Blackstone EH. Persistent effect of red cell transfusion on health-related quality of life after cardiac surgery. Ann Thorac Surg. 2006;82:13-20.

19. Brevig J, McDonald J, Zelinka ES, Gallagher T, Jin R, Grunkemeier GL. Blood transfusion reduction in cardiac surgery: multidisciplinary approach at a community hospital. Ann Thorac Surg. 2009;87:532-9.

20. Helm RE, Rosengart TK, Gomez M, Klemperer JD, DeBois WJ, Velasco F, et al. Comprehensive multimodality blood conservation: 100 consecutive CABG operations without transfusion. Ann Thorac Surg. 1998;65:125-36.

21. Moskowitz DM, Klein JJ, Shander A, Cousineau KM, Goldweit RS, Bodian C, et al. Predictors of transfusion requirements for cardiac surgical procedures at a blood conservation center. Ann Thorac Surg. 2004;77:626-34

22. Moskowitz DM, McCullough JN, Shander A, Klein JJ, Bodian CA, Goldweit RS, et al. The impact of blood conservation on outcomes in cardiac surgery: is it safe and effective? Ann Thorac Surg. 2010;90:451-8.

23. Ovrum E, Holen EA, Abdelnoor M, Oystese R. Conventional blood conservation techniques in 500 consecutive coronary artery bypass operations. Ann Thorac Surg. 1991;52:500-5.

24. Ngaage DL. Off-pump coronary artery bypass grafting: the myth, the logic and the science. Eur J Cardiothorac Surg. 2003;24:557-70.

25. Bracey AW, Radovancevic R, Riggs SA, Houston S, Cozart H, Vaughn WK, et al. Lowering the hemoglobin threshold for transfusion in coronary artery bypass procedures: effect on patient outcome. Transfusion. 1999;39:1070-7.

26. DeAnda A Jr, Baker KM, Roseff SD, Green JA, McCarthy H, Aron T, et al. Developing a blood conservation program in cardiac surgery. Am J Med Qual. 2006; 21:230-7.

27. Ferraris VA, Ferraris SP, Saha SP, Hessel EA 2nd, Haan CK, Royston BD, et al. Perioperative blood transfusion and blood conservation in cardiac surgery: the Society of Thoracic Surgeons and The Society of Cardiovascular Anesthesiologists clinical practice guideline. Ann Thorac Surg. 2007;83(5 Suppl):S27-86.

28. Hebert PC, Wells G, Blajchman MA, Marshall J, Martin C, Pagliarello G, et al. A multicenter, randomized, controlled clinical trial of transfusion requirements in critical care. Transfusion Requirements in Critical Care Investigators, Canadian Critical Care Trials Group. N Engl J Med. 1999;340:409-17.

29. Whitson BA, Huddleston SJ, Savik K, Shumway SJ. Bloodless cardiac surgery is associated with decreased morbidity and mortality. J Card Surg. 2007;22:373-8.

30. Stokes ME, Ye X, Shah M, Mercaldi K, Reynolds MW, Rupnow MF, et al. Impact of bleeding-related complications and/or blood product transfusions on hospital costs in inpatient surgical patients. BMC Health Serv Res. 2011;11:135.

\section{Discussion}

Dr Edward Verrier (Seattle, Wash). Drs Lazar, McGiffin, and LaPar, thank you for the opportunity to discuss this well-presented analysis of outcomes and costs after the implementation of a multidisciplinary blood conservation program incorporating 14,000 patients in 17 different cardiac surgery programs in the Commonwealth of Virginia. The Virginia Cardiac Surgery Quality Initiative should be commended for their organization, structure, database, and priorities.
The study looks at nonemergent isolated CABG patients over 2 time periods-2006 to 2008 and 2008 through 2010. Strict blood transfusion triggers were implemented intraoperatively and in the surgical intensive care unit, and looked at short-term outcome variables and detailed analysis of costs with the introduction of such a blood conservation program.

The conclusions of improved outcomes are not new as carefully pointed out in the manuscript. The details of the cost improvement with such a program are the most significant contribution of this effort. In addition, the scope of involvement over an entire state reveals the accomplishments that can be achieved with excellent organization, priority, commitment, and leadership.

Dr Bruce Spiess, one of the authors of this effort, and I shared similar improvements in outcomes with the initiation of such a blood conservation program at the University of Washington more than 20 years ago, and we strictly follow that transfusion protocol today.

The authors note the limitations of the study in this manuscript. The study is retrospective, spans 2 sequential time periods, does not account for improvements in blood banking or critical care services, miscoding, and certainly could be influenced by increased awareness and better discipline by the entire surgical, anesthesia, perfusion, and critical care teams.

My first question relates to cause and effect. Did the authors carefully look through the STS database over the same time period to see if improvements in outcomes for isolated elective 2-vessel CABG also improved nationally in the large database in programs without the initiation of such a blood transfusion conservation protocol? I suspect a modest improvement in outcomes noted by the authors might also have been seen over that time frame, as reflected in that large database.

Dr LaPar. Thank you very much for your question, and I think that you bring up some very important points, the first being that when we are comparing data within a region or state, it is very important to compare that data with national trends and outcomes. Nationally, we have seen CABG outcomes improve over time, and certainly these trends and the influence of a time bias, for example, may influence to a small extent the results of this study.

In these analyses, we did not include a subanalysis of CABG outcomes by individual center or program to further determine the effect of improving CABG outcomes over time. This is an interesting point and one that deserves further investigation.

Dr Verrier. My second question relates to the question of transfusion as a marker of injury versus transfusion as an immunomodulator. It is similar to the discussion of acute renal failure in outcomes. We know that patients with acute renal failure do worse, and the question is, is that because they have acute renal failure, or is the renal failure a reflection of the complexity of the disease?

I realize this study was risk adjusted, but in every discussion in the cardiothoracic literature or trauma literature, the question of whether transfusion is a double-edged sword that we have come to understand, is that true or not? Did the authors get any significant scientific insight related to cytokine production, viral vectors, or immunologic mediators that caused this adverse effect on outcomes such as transfusion-related pulmonary toxicity or acute tubular necrosis?

Dr LaPar. Unfortunately, because of the de-identified nature of the VSCQI data registry, we were confined in these analyses to 
data currently captured by the STS data collection forms. Although I agree that the systemic and inflammatory markers you mentioned may, in fact, provide further information on the effects and or benefits of blood product use or conservation, we were unable to analyze such data. Rather, we are able to gain some insight into these effects in our comparison of the clinical manifestations of these processes such as the incidence of renal insufficiency or prolonged mechanical ventilation as we have in our analysis. These differences remain hypothesis generating though, and I agree that it would be interesting to further investigate such effects of immunomodulation at the single institution level. This is a question that we will take back to our institution to look at in the future.

Dr Verrier. I certainly enjoyed the opportunity to hear this excellent presentation and believe this will be an important contribution to our literature. I hope that it is now extended, based on this experience at the statewide level, to the national level because the implications there at the national level, both on the cost analysis and outcomes, would be significant. Thank you for the opportunity.

Dr LaPar. Thank you.

Dr David McGiffin (Birmingham, Ala). I do not want to take anything away from your study, because I agree with your conclusions, but I just wanted to make an observation that when I look at that table of the number of outcomes from the preguideline to the postguideline eras, the differences are very, very small-for example, prolonged ventilation, $4.7 \%$ to $4.4 \%$. And we have to be careful we that we do not overemphasize the very small differences that may be statistically significant but of very questionable biologic importance, because there is no difference that is not small enough that we cannot transform into a significant $P$ value by having sufficient numbers.

Dr LaPar. Certainly the effect of a large sample size, as in our study, allows small differences to achieve statistical significance. However, some of the most significant limitations of former series evaluating the effects of blood product use in cardiac surgery are the inclusion of smaller patient samples from single institutions that limits the statistical power of their analyses and the generalizability of their results. Our study addresses these concerns with a large, multi-institutional cohort of patients and outcomes.

Dr Paul Kurlansky (Miami, Fla). I would like to expand on the first point that Dr Verrier brought up, which is the effect of time on behavior. Virginia has done a magnificent job in bringing people together and trying to work together on quality initiatives. However, as I understand it, there is tremendous variability on the actual application or implementation of various guidelines that are recommended by the collaborative. I was wondering if you have any mechanism to track the compliance with the guidelines amongst the various 17 different centers and whether compliance was in any way associated with outcome as a way to guard against the post hoc ergo propter hoc influence of time?

Dr LaPar. That is a great point, and I think it is important to recognize that in this study there was some variability between participating centers in the enforcement of the blood transfusion guidelines once they were agreed upon. With respect to our analyses, the effect of this variability would likely bias the results toward the null. To date, our organization has not implemented a particular mechanism to track center compliance. Such a process would be helpful, however, and is something we can discuss for future comparisons.

As you mentioned, one of the greatest strengths of this study is that it shows the ability to organize a group of centers, bring them together to develop a group of guidelines, and to implement the guidelines to achieve not only improved clinical outcomes but also financial benefits as well. And our demonstration of a \$49 million reduction in overall total hospitalization costs during a 2-year period in the Commonwealth of Virginia is extremely important. It would be great if we could capture the financial benefits of such an initiative at the nationwide level.

More importantly, we would like to emphasize that we do know that within hospitals that did very well with the guideline compliance that these hospitals actively involved the individual caregiver responsible for carrying out the guideline specifics. These individuals included cardiac surgical trainees and fellows, nurse practitioners, or physicians assistants, depending on the center. To be successful, it is important to involve these individual early in the implementation of the guidelines and then provide them consistent and frequent feedback on impact of the initiative. When this happens, it serves to reinforce guideline compliance.

Dr Harold Lazar (Boston, Mass). Can you tell us what the difference was in the incidence of the use of Plavix between the groups? And also can you tell us a little bit about the cardiopulmonary bypass techniques? Did they use heparin-bonded circuits? Was miniature circuitry used? Can you tell us anything about those techniques between the time periods?

Dr LaPar. In our patient cohort, the use of Plavix was higher in the postguideline era compared with the preguidelines era. And, I am sorry, I could not exactly hear your question related to cardiopulmonary bypass.

Dr Lazar. The techniques of cardiopulmonary bypass, whether or not you used heparin-bonded circuitry, whether you used cardiotomies for your pumps.

Dr LaPar. Those are important factors, and we did not have that data available to analyze. The impact of these techniques would certainly be something that we can look at in the future as this is an area of research that we want to continue to investigate within our organization. 